\title{
Holographic entanglement entropy decomposition in an anisotropic gauge theory
}

\author{
M. Rahimi ${ }^{*}$ and M. Ali-Akbari ${ }^{\dagger}$ \\ Department of Physics, Shahid Beheshti University G.C., Evin, Tehran 19839, Iran
}

(Received 7 March 2018; published 2 July 2018)

\begin{abstract}
We study holographic entanglement entropy in spatially anisotropic field theory. We observe that for the background considered in this paper, to a good approximation, the holographic entanglement entropy can be decomposed into two terms. One of them is the entanglement entropy of the isotropic field theory at a fixed temperature and the other term is only a function of the anisotropy parameter. Moreover, for large enough values of the anisotropy parameter, our numerical results indicate that the entanglement entropy in the perpendicular direction to the anisotropic direction is greater than the parallel case.
\end{abstract}

DOI: 10.1103/PhysRevD.98.026004

\section{INTRODUCTION}

During the past two decades, gauge-gravity duality received a lot of interest for explaining the physics of strongly coupled field theories as a new framework. Since standard methods such as perturbative expansion are not applicable due to a large coupling constant, gauge-gravity duality plays an important role in this area of physics. This duality states that a strongly coupled gauge field theory in $d$-dimensional space-time corresponds to a classical gravity in the $d+1$ dimensional background [1]. According to the gaugegravity duality, the field theory is living on the boundary of the background. All parameters, fields, and processes in the gauge theory are then translated into an appropriate equivalent on the gravity side. Up to now, this duality has been frequently applied and reveals a lot of valuable information about strongly coupled field theories. For a good review with an extensive list of references, see [2].

For describing the various properties of strongly coupled gauge theories, this duality usually proposes applicable and simple prescriptions in gravity theory. In other words, although explaining different properties may not seem simple from the field theory perspective, its gravitational description is generally more tractable. As an example, there is a very simple prescription, first introduced in [3], in order to calculate the entanglement entropy in the field theories with a holographic dual. The entanglement entropy is analytically calculated in two-dimensional conformal

\footnotetext{
me_rahimi@sbu.ac.ir

m_aliakbari@sbu.ac.ir
}

Published by the American Physical Society under the terms of the Creative Commons Attribution 4.0 International license. Further distribution of this work must maintain attribution to the author(s) and the published article's title, journal citation, and DOI. Funded by SCOAP ${ }^{3}$. field theory, and its generalization to higher dimensions is not obvious. However, as we will see in the next section, its gravitational dual is simple and can be generalized to arbitrary dimensions. This conjectured formula does indeed satisfy many nontrivial relations known in quantum information theory.

In this paper, using the holographic description of the entanglement entropy, we would like to investigate the effect of the anisotropy parameter $a$ on the entanglement entropy in an anisotropic thermal gauge field theory. Here, "anisotropic" refers to spatially anisotropic systems or equivalently, systems where the pressure in one direction, for example $z$, is different from the others. For this reason, we will work with the anisotropic background introduced in [4]. Then we consider the entangling length in the anisotropic direction $z$, called the parallel case, or in the transverse directions, i.e., perpendicular to $z$, the perpendicular case. According to our numerical outcomes, the main findings can be summarized as follows:

(i) At a fixed temperature $T$, the entanglement entropy for the perpendicular case is greater than the parallel case, that is $S_{A}^{\perp}>S_{A}^{\|}$for $a>2 T$. The same behavior is also true at a fixed entropy density $s$ for $a>2 s^{1 / 3}$. It is important to notice that this behavior does not persist in the high temperature limit, i.e., $T \gg a$.

(ii) At a fixed temperature, for both the parallel and perpendicular cases, to a good approximation, the entanglement entropy can be decomposed into two terms: one of them is the entanglement entropy of the isotropic field theory at a finite temperature, and the other one is independent of the temperature and depends only on the anisotropy parameter. This statement is also true when the entropy density is kept fixed. Interestingly, this decomposition can be done in the high temperature limit, too. 
(iii) In the desired range of an anisotropy parameter, the only term which $a$ depends on, the anisotropy parameter, is proportional to $k_{1} a^{4}-k_{2} a^{2}\left(-n_{1} a^{2}+\right.$ $n_{2} a^{4}$ ) for a perpendicular (parallel) case, where $k_{1}$, $k_{2}, n_{1}$, and $n_{2}$ are positive numbers. Independent of temperature and entropy density which are kept fixed, the coefficients $k_{1}$ and $k_{2}\left(n_{1}\right.$ and $\left.n_{2}\right)$ are the same, interestingly.

(iv) Making the anisotropy parameter larger, the deviation of an anisotropic entanglement entropy form the isotropic one becomes greater when the temperature or entropy density is kept fixed.

(v) We checked and believed that all results reported in this paper exist for any acceptable entangling length.

\section{REVIEW ON (HOLOGRAPHIC) ENTANGLEMENT ENTROPY}

A well-known nonlocal observable in the quantum field theory is the entanglement entropy [5]. Consider a quantum field theory whose state is described by the density matrix $\rho$. The entanglement entropy of a spatial subregion $A$, with complement $B$, denotes how much entanglement exists between $A$ and $B$, and it is given by

$$
S_{A}=-\operatorname{Tr}\left(\rho_{A} \log \rho_{A}\right),
$$

where $\rho_{A}=\operatorname{Tr}_{B}(\rho)$ is a reduced matrix and obtained by tracing over the degrees of freedom in the region $B$. The entanglement entropy of $A$ shows the amount of information lost when an observer is limited to the subregion $A$. Moreover, it has a main divergency which is proportional to the area of the subregion $A$ for a space-time dimension greater than 2. Although calculating the entanglement entropy is normally difficult, it has a famous and simple description in the context of the gauge-gravity duality. In fact, Ryo and Takayanagi first proposed in [3] that the entanglement entropy can be computed from

$$
S_{A}=\frac{\operatorname{Area}\left(\gamma_{A}\right)}{4 G_{N}^{d+2}},
$$

where $G_{N}^{d+2}$ is the $d$-dimensional Newton constant. $\gamma_{A}$ is a codimension-2 minimal surface whose boundary $\partial \gamma_{A}$ coincides with the boundary of the subregion $A$ on the boundary of the bulk where the quantum field theory lives, i.e., $\partial \gamma_{A}=\partial A$. This proposal received a lot of interest during the last decade and passed several nontrivial checks known in the quantum field theory. For more details, we refer the reader to [6]. In order to calculate the holographic entanglement entropy, we start with a $d+2$-dimensional background

$d s^{2}=-f_{1}(u) d t^{2}+f_{2}(u) d u^{2}+f_{3}(u) d z^{2}+f_{4}(u) d \vec{x}^{2}$,

where $u$ is radial direction and the boundary is located at $u=0 . f_{1}, \ldots, f_{4}$ are arbitrary functions and depend only on the radial direction. $\left(t, z, \vec{x}=x_{1}, \ldots, x_{d-1}\right)$ represent $d+1$ dimensional boundary coordinates where the field theory lives. We also demand that the background approaches $\mathrm{AdS}_{d+2}$ with an asymptotical radius of one. As it is clearly seen, since generally $f_{3} \neq f_{4}$, the above background is anisotropic along the gauge theory directions, and hereafter, we call $z$ the anisotropic coordinate. $f_{1}$ and $f_{2}$ are blacking factors that vanish at the position of the horizon, i.e., $f_{1}\left(u=u_{h}\right)=f_{2}^{-1}\left(u=u_{h}\right)=0$, and therefore, the above metric is a black hole solution. According to the gauge-gravity duality, the Hawking temperature of the black hole is identified with the temperature of the field theory. We now consider the simplest shape for the boundary entangling region $A$, which is a rectangular shape with one dimension of the length $l$ in $z$ (parallel to the anisotropic coordinate) or $x \in\left(x_{1}, \ldots, x_{d-1}\right)$ (perpendicular to the anisotropic coordinate) direction at a constant time slice and assume all other coordinates have infinite width, see Fig. 1. Note that since there is a rotational symmetry in $\vec{x}$ directions, there is no difference between them. The recipe (2) states that the entanglement entropy between the regions $A$ and its complement $B$ is proportional to the minimal surface $\gamma_{A}$, and it can be then obtained from

$$
S_{A}=\frac{1}{4 G_{N}^{d+2}} \int d^{d} \sigma \sqrt{\operatorname{det} g_{a b}},
$$

where $g_{a b}$ is an induced metric on $\gamma_{A}$ and is defined as $g_{a b}=G_{M N} \partial_{a} X^{M} \partial_{b} X^{N} . X^{M_{\mathrm{S}}}$ are bulk coordinates introduced in (3), and $\sigma^{a}$ s denote coordinates on the $\gamma_{A}$, and we thus have $X^{M}\left(\sigma^{a}\right)$. In the perpendicular case, using the static gauge, i.e., $\left(\sigma^{1}, \ldots, \sigma^{d}\right) \equiv\left(z, x^{1}, \ldots, x^{d-1}\right)$, and due to the translational symmetry in the all directions in the background except $x \equiv x^{d-1}$, the shape of the minimal surface corresponding to the rectangular strip is described by $u(x)$, see Fig. 1. Similarly, for the parallel case, the minimal surface can be described by $u(z)$. In the following, using the gaugegravity duality, we proceed our calculations to obtain the entanglement entropy for these two cases.

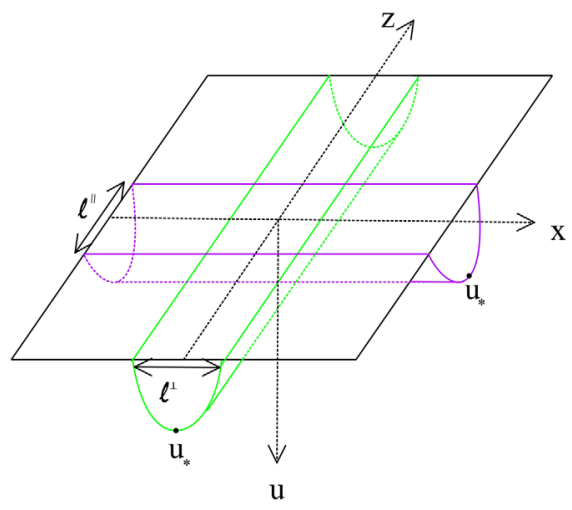

FIG. 1. Minimal surfaces in the anisotropic background. 
(i) Perpendicular case: In this case, we have $u(x)$, and after a simple calculation, the holographic entanglement entropy (4) leads to

$$
S_{A}^{\perp}=\frac{V_{d-1}}{4 G_{N}^{d+2}} \int_{-\frac{1}{2}}^{\frac{l}{2}} d x \sqrt{f_{3}(u) f_{4}^{d-1}(u)+f_{2}(u) f_{4}^{d-2}(u) f_{3}(u) u^{\prime 2}},
$$

where $u^{\prime}=\frac{d u}{d x}$ and $V_{d-1}$ is the volume of the isotropic transverse coordinates. Since the above equation does not depend explicitly on the $x$, a constant of motion can be easily found as

$$
\frac{f_{3}(u) f_{4}^{d-1}(u)}{\sqrt{f_{3}(u) f_{4}^{d-1}(u)+f_{2}(u) f_{4}^{d-2}(u) f_{3}(u) u^{\prime 2}}}=f_{3}\left(u_{*}\right) f_{4}^{d-1}\left(u_{*}\right),
$$

where $u_{*}$ is the turning point at which $u^{\prime}(x)=0$ and is located at $x=0$ by symmetry. It then turns out that

$$
u^{\prime}=\left(\frac{f_{4}}{f_{2}}\left(\frac{f_{3} f_{4}^{d-1}}{f_{3 *} f_{4 *}^{d-1}}-1\right)\right)^{\frac{1}{2}}
$$

where $f_{3 *}=f_{3}\left(u_{*}\right)$ and so on. From the above equation, one gets

$$
l^{\perp}=2 \int_{0}^{u_{*}} d u\left(\frac{f_{4}}{f_{2}}\left(\frac{f_{3} f_{4}^{d-1}}{f_{3 *} f_{4 *}^{d-1}}-1\right)\right)^{-\frac{1}{2}}
$$

Finally, by substituting (7) into (5), the holographic entanglement can be obtained as

$$
S_{A}^{\perp}=\frac{V_{d-1}}{2 G_{N}^{d+2}} \int_{0}^{u_{*}} d u \frac{\sqrt{f_{2}} f_{4}^{d-\frac{3}{2}} f_{3}}{\sqrt{f_{3} f_{4}^{d-1}-f_{3 *} f_{4 *}^{d-1}}}
$$

(ii) Parallel case: In this case, as it was already explained, we have $u(z)$. Similar to the perpendicular case, the entanglement entropy and entangling length $l^{\|}$can be found

$$
\begin{aligned}
& S_{A}^{\|}=\frac{V_{d-1}}{2 G_{N}^{d+2}} \int_{0}^{u_{*}} d u \frac{\sqrt{f_{2} f_{3}} f_{4}^{d-1}}{\sqrt{f_{3} f_{4}^{d-1}-f_{3 *} f_{4 *}^{d-1}}}, \\
& l^{\|}=2 \int_{0}^{u_{*}} d u\left(\frac{f_{3}}{f_{2}}\left(\frac{f_{3} f_{4}^{d-1}}{f_{3 *} f_{4 *}^{d-1}}-1\right)\right)^{-\frac{1}{2}} .
\end{aligned}
$$

A specific solution of (3) is an isotropic black hole background which is given by

$$
\begin{aligned}
u^{2} f_{1}(u) & =u^{-2} f_{2}^{-1}(u)=1-\frac{u^{4}}{u_{h}^{4}}, \\
f_{3}(u) & =f_{4}(u)=\frac{1}{u^{2}}
\end{aligned}
$$

Obviously, for the above solution, we get $S_{A}^{0}=S_{A}^{\perp}=S_{A}^{\|}$. As it is well-known, the entanglement entropies $S_{A}^{\perp}$ and $S_{A}^{\|}$ are divergent, and the divergent term is proportional to the area of the subregion $A$. In order to find finite entanglement entropies, we define the following functions:

$$
\begin{aligned}
& \Delta S^{\perp}=\frac{G_{N}^{d+2}}{V_{d-1}}\left(S_{A}^{\perp}-S_{A}^{0}\right), \\
& \Delta S^{\|}=\frac{G_{N}^{d+2}}{V_{d-1}}\left(S_{A}^{\|}-S_{A}^{0}\right) .
\end{aligned}
$$

where $\Delta S^{\perp}$ and $\Delta S^{\|}$are now finite. We emphasize here that these two quantities represent the difference between entanglement entropies in an anisotropic and isotropic background which will be computed at a fixed temperature or fixed entropy density. More specifically, they provide noteworthy information about the effect of an anisotropy parameter on the entanglement entropies, as we will see in the next section.

\section{NUMERICAL RESULTS}

The background we are interested in is an anisotropic solution of the IIB string theory introduced in [4], and different aspects of this solution have been illustrated in the literature, for instance, see [7]. Regarding (3), the anisotropic metric with $d=3$ is

$$
\begin{aligned}
& f_{1}(u)=\mathcal{F} \mathcal{B} u^{-2}, \quad f_{2}(u)=\mathcal{F}^{-1} u^{-2}, \\
& f_{3}(u)=\mathcal{H} u^{-2}, \quad f_{4}(u)=u^{-2} .
\end{aligned}
$$

The functions $\mathcal{H}, \mathcal{F}$, and $\mathcal{B}$ depend only on the radial direction. In terms of the Dilaton field, they are

$$
\begin{aligned}
\mathcal{H} & =e^{-\phi}, \\
\mathcal{F} & =\frac{e^{-\frac{1}{2} \phi}\left[a^{2} e^{\frac{1}{2} \phi}\left(4 u+u^{2} \phi^{\prime}\right)+16 \phi^{\prime}\right]}{4\left(\phi^{\prime}+u \phi^{\prime \prime}\right)}, \\
\frac{\mathcal{B}^{\prime}}{\mathcal{B}} & =\frac{1}{24+10 u \phi^{\prime}}\left(24 \phi^{\prime}-9 u \phi^{\prime 2}+20 u \phi^{\prime \prime}\right),
\end{aligned}
$$

where the Dilaton field satisfies the following third-order equation: 

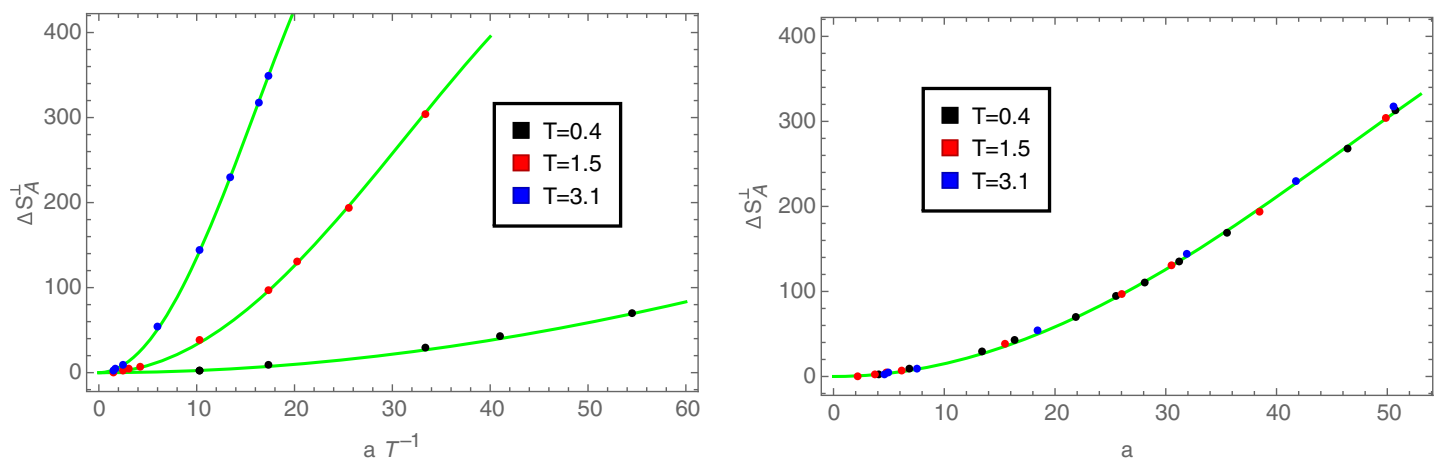

FIG. 2. $\Delta S_{A}^{\perp}$ in terms of (a) $\frac{a}{T}$ (left) and (b) $a$ (right) at fixed temperatures for $l=0.1$. The green curves show the fitted functions (16) and (17) in the left and right figure, respectively.

$$
\begin{aligned}
& \frac{256 \phi^{\prime} \phi^{\prime \prime}-16 \phi^{\prime 3}\left(7 u \phi^{\prime}+32\right)}{u a^{2} e^{\frac{7 \phi}{2}}\left(u \phi^{\prime}+4\right)+16 \phi^{\prime}}+\frac{\phi^{\prime}}{u\left(5 u \phi^{\prime}+12\right)\left(u \phi^{\prime \prime}+\phi^{\prime}\right)} \\
& \times\left[13 u^{3} \phi^{\prime 4}+8 u\left(11 u^{2} \phi^{\prime 2}-60 \phi^{\prime \prime}-12 u \phi^{\prime \prime \prime}\right)\right. \\
& +u^{2} \phi^{\prime 3}\left(13 u^{2} \phi^{\prime \prime}+96\right)+2 u \phi^{\prime 2}\left(-5 u^{3} \phi^{\prime \prime \prime}+53 u^{2} \phi^{\prime \prime}+36\right) \\
& \left.+\phi^{\prime}\left(30 u^{4} \phi^{\prime \prime 2}-64 u^{3} \phi^{\prime \prime \prime}-288+32 u^{2} \phi^{\prime \prime}\right)\right]=0 .
\end{aligned}
$$

Note that the solution also contains a self dual fiveform field. The horizon is located at $u=u_{h}$, where $\mathcal{F}\left(u_{h}\right)=0$. The Hawking temperature and entropy density per unit volume are given by [4]

$$
\begin{aligned}
& T=-\frac{1}{4 \pi} \mathcal{F}^{\prime}\left(u_{h}\right) \sqrt{\mathcal{B}\left(u_{h}\right)}, \\
& s=\frac{\pi^{2} N_{c}^{2}}{2} \frac{e^{-\frac{5}{4} \phi_{h}}}{\pi^{3} u_{h}^{3}},
\end{aligned}
$$

where $\phi_{h}$ is the value of the Dilaton field at the horizon and $N_{c}$ denotes the number of the color in the gauge theory. In this solution, the nonzero anisotropy parameter in the field theory is introduced by an axion field in the background corresponding to a position-dependent $\theta$ term or, more precisely, $\theta=a z$. The $\theta$ term breaks the original isotropy of the system and forces the system into an anisotropic equilibrium state. It also leads to a nonzero conformal anomaly, meaning that the trace of the energy-momentum tensor is no longer zero and is proportional to the anisotropy parameter $a$ which has a dimension of energy. In other words, in the field theory corresponding to the geometry (13), the pressure in the $z$ and $\vec{x}$ directions are not equal. For more details, see [4]. After finding the solution (13) numerically, our main task in the following is to understand how the anisotropy parameter modifies the entanglement entropies $S_{A}^{\perp}$ and $S_{A}^{\|}$.

\section{A. Perpendicular case}

(1) Fixed temperature: In Fig. 2(a), we have plotted $\Delta S^{\perp}$ in terms of $a / T$ for three different values of temperature. Evidently, $\Delta S^{\perp}>0$ meaning that
$S_{A}^{\perp}>S_{A}^{0}$. Furthermore, the larger the anisotropy parameter, the greater the deviation. We also observe that our numerical outcomes, the blue, red and black points in Fig. 2(a), are fitted with

$\Delta S_{A}^{\perp}(a, T)=K_{1}(T)\left(\frac{a}{T}\right)^{2}-K_{2}(T)\left(\frac{a}{T}\right)^{4}$,

where the values of functions $K_{1}(T)$ and $K_{2}(T)$ are listed in Table I. Another valuable result comes out when one plots $\Delta S^{\perp}$ in terms of an anisotropy parameter $a$ instead of $a / T$, see Fig. 2(b). Surprisingly, it shows that the value of $\Delta S^{\perp}$, for various values of temperature, depends only on the anisotropy parameter and can be fitted with

$$
\Delta S^{\perp}(a)=k_{1} a^{2}-k_{2} a^{4},
$$

where $k_{1}$ and $k_{2}$ are positive constants and their values are also presented in Table I. In fact, (17) indicates that, to a good approximation, $\Delta S^{\perp}$ is independent of temperature and the coefficients $k_{1}$ and $k_{2}$ are enough to compute the value of $\Delta S^{\perp}$ for a given anisotropy parameter. However, the temperature dependence explicitly appears in (16), and therefore, these two equations, that is (16) and (17), force us to consider $K_{1}(T)=k_{1} T^{2}$ and $K_{2}(T)=$ $k_{2} T^{4}$. Before closing this part, a few comments are in order.

(a) Although the values of $k_{1}$ or $k_{2}$ are not exactly the same for the three values of the temperature, one needs a much greater precision in the

TABLE I. Coefficients of (16) and (17).

\begin{tabular}{lcccc}
\hline \hline$T$ & $K_{1}$ & $K_{2}$ & $k_{1}$ & $k_{2}$ \\
\hline 0.4 & 0.024 & $3.27 \times 10^{-7}$ & 0.150 & $1.145 \times 10^{-5}$ \\
1.5 & 0.337 & $5.63 \times 10^{-5}$ & 0.150 & $1.112 \times 10^{-5}$ \\
3.1 & 1.445 & $9.42 \times 10^{-4}$ & 0.152 & $1.115 \times 10^{-5}$ \\
\hline \hline
\end{tabular}



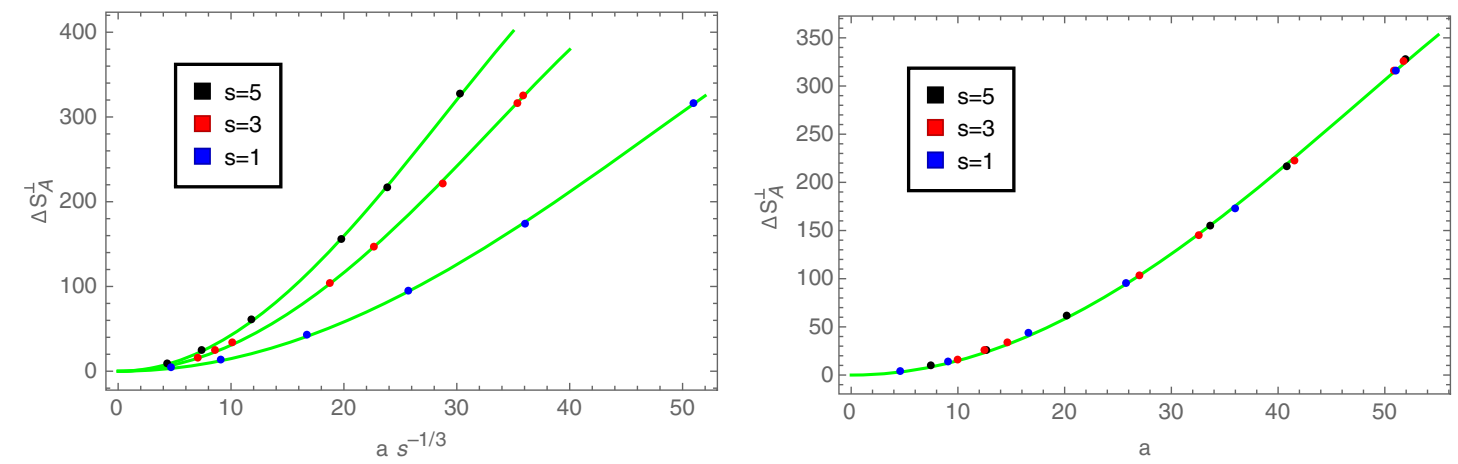

FIG. 3. $\Delta S_{A}^{\perp}$ in terms of (a) $a s^{-1 / 3}$ (left) and (b) $a$ (right) at a fixed entropy density for $l=0.1$. The green curves show the fitted functions (21) (left) and (17) (right).

numerical calculation to approach closer to the same value. In other words, the differences among the values of $k_{1}$ or $k_{2}$ would be more negligible with a greater precision.

(b) The value of $K_{2}$ is always too small compared to $K_{1}$. Thus, the second term in (16), or equivalently, in (17), does not change the value of the $\Delta S^{\perp}$ substantially for small values of an anisotropy parameter. In other words, if one ignores this term, the maximum relative error, i.e., $\left|\frac{\Delta S^{\perp}-\left(\Delta S^{\perp}\right)_{k_{2}=0}}{\Delta S^{\perp}}\right|$, is about 0.23 for $a=50$.

(c) Since the trace of the energy-momentum tensor is proportional to an anisotropy parameter [4], one can conclude that the entanglement entropy is larger when the conformal symmetry is highly broken.

(d) From (12a) and (17), it turns out that

$S_{A}^{\perp}(a, T)=\frac{V_{d-1}}{G_{N}^{d+2}}\left(k_{1} a^{2}-k_{2} a^{4}\right)+S_{A}^{0}(T)$.

The first (second) term in the above equation is only a function of an anisotropy parameter (temperature). Thus, our results propose that the entanglement entropy in the anisotropic background we consider in this paper can be decomposed as

$$
S_{A}^{\perp}(a, T)=\hat{S}_{A}(a)+S_{A}^{0}(T),
$$

where

$$
\hat{S}_{A}(a)=\frac{V_{d-1}}{G_{N}^{d+2}}\left(k_{1} a^{2}-k_{2} a^{4}\right) .
$$

In fact, the anisotropy parameter and temperature do not communicate with each other.

(e) The last point, perhaps the more important one, is that all above numerical outcomes are reliable in the range of $4 T<a<50$.
(2) Fixed entropy density: Instead of temperature, the entropy density can be kept fixed, too. In order to have a dimensionless parameter, we introduce $a s^{-1 / 3}$, where $s \equiv s / N_{c}^{2}$. We then plot the entanglement entropy in terms of this new dimensionless quantity, and the results are shown in Fig. 3. Similar to the case of a fixed temperature, we observe that the numerical data are fitted with

$S_{A}^{\perp}(a, s)=\hat{K}_{1}(s)\left(a s^{-1 / 3}\right)^{2}-\hat{K}_{2}(s)\left(a s^{-1 / 3}\right)^{4}$,

where the values of $\hat{K}_{1}$ and $\hat{K}_{2}$ are listed in Table II. Interestingly, although $\hat{K}_{1}$ and $\hat{K}_{2}$ are not equal to $K_{1}$ and $K_{2}$, numerical results in the Fig. 3(b) reveal that the entanglement entropy still satisfies (17) for different values of fixed entropy density with unchanged coefficients $k_{1}$ and $k_{2}$. Notice that in this case, the temperature appeared in (19) can be found in terms of an anisotropy parameter and entropy density using (15). As a matter of fact, for given values of an entropy density and anisotropy parameter, one can find the corresponding temperature. We find out that the entanglement entropy, given by (17) or equivalently (19), is independent of the values of the temperature and entropy density in the desired range of an anisotropy parameter. Similar comments argued in the case of a fixed temperature can be also discussed in the current case, and we do not repeat them here.

Up to now, according to our numerical results, we claim that $\Delta S_{A}^{\perp}$ is only a function of the anisotropy parameter in the background we consider. As a cross-check, in Fig. 4, the

TABLE II. Coefficients of (17) and (21).

\begin{tabular}{ccccc}
\hline \hline$s$ & $\hat{K}_{1}$ & $\hat{K}_{2}$ & $k_{1}$ & $k_{2}$ \\
\hline 1 & 0.150 & $1.090 \times 10^{-5}$ & 0.150 & $1.090 \times 10^{-5}$ \\
3 & 0.310 & $4.526 \times 10^{-5}$ & 0.149 & $1.046 \times 10^{-5}$ \\
5 & 0.434 & $4.526 \times 10^{-5}$ & 0.148 & $1.013 \times 10^{-5}$ \\
\hline \hline
\end{tabular}




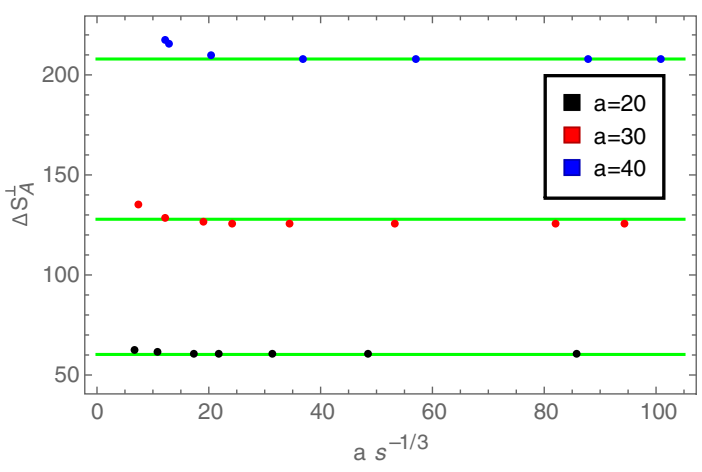

FIG. 4. $\Delta S_{A}^{\perp}$ as a function of (a) $a s^{-1 / 3}$

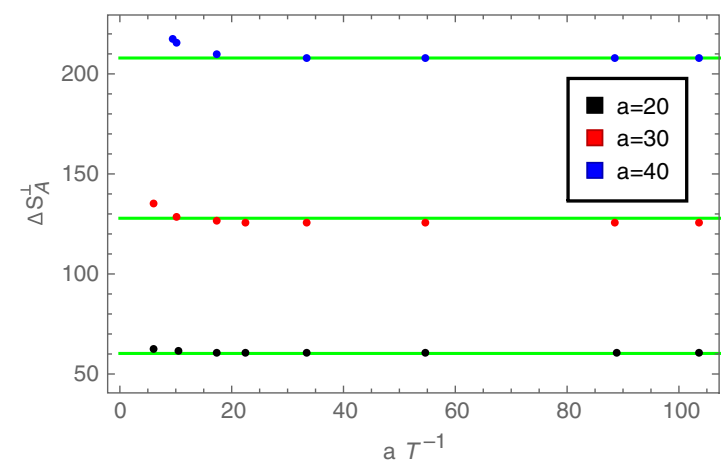

3 (right) and (b) $\frac{a}{T}$ (left) for $l=0.1$. entanglement entropy is plotted in terms of the temperature and entropy density for fixed values of anisotropy parameters. As it is clearly seen, for large enough values of the anisotropy parameter, that is $a>2 s^{1 / 3}$ or $a>2 T$, the entanglement entropy is given by (17) to a good approximation. In fact, these figures prove our previous results and claim.

\section{B. Parallel case}

In this section, we consider the case in which the entangling length is parallel to the anisotropy direction $z$. Figure 5 shows our results. The main difference between the parallel and perpendicular case is that in the parallel case, $\Delta S^{\|}<0$, meaning that $S_{A}^{\|}<S_{A}^{0}$, opposite to the perpendicular case for which $S_{A}^{\perp}>S_{A}^{0}$. A few of significant outcomes can be summarized as follows:

(i) In the desired range of the anisotropy parameter, in both cases, the value of the parallel entanglement entropy $S_{A}^{\|}$is always smaller than $S_{A}^{0}$ when the temperature or entropy density is kept fixed.

(ii) For a larger anisotropy parameter, the deviation of $S_{A}^{\|}$from the isotropic entanglement entropy becomes greater.

(iii) The entanglement entropy in the parallel case can be decomposed as

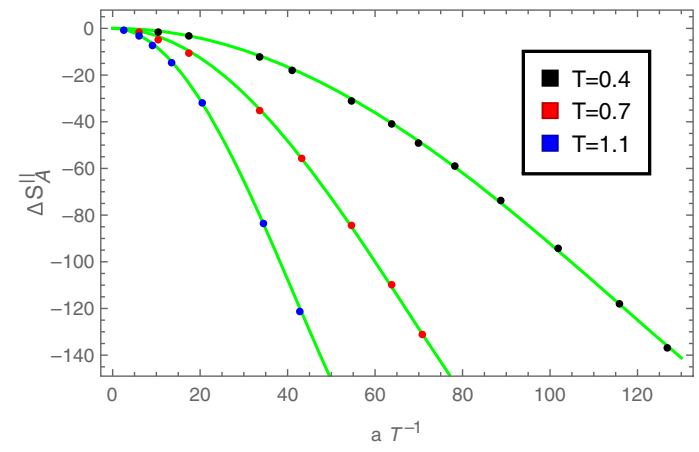

$$
S_{A}^{\|}(a, T)=\tilde{S}_{A}(a)+S_{A}^{0}(T)
$$

where $\tilde{S}_{A}(a)=\frac{V_{d-1}}{G_{N}^{d+2}}\left(-n_{1} a^{2}+n_{2} a^{4}\right), \quad n_{1}=0.065$, and $n_{2}=5 \times 10^{-6}$ for a fixed value of temperature or entropy density cases.

In this section, our most important outcome is that for a large enough $a / T$ (or $a s^{-1 / 3}$ ), the entanglement entropy in the parallel and perpendicular direction can be decomposed into a temperature dependent term, which is the entanglement entropy of the isotropic field theory at a finite temperature, and an anisotropic part which is independent of the temperature (or entropy density) to a good approximation. As we will see in the next section, this behavior persists in the high temperature limit, too.

\section{HIGH TEMPERATURE LIMIT}

The analytical solution for (13) has been introduced in [4] in the high temperature limit, that is $T \gg a$. The metrics components are given by

$$
\begin{aligned}
\mathcal{F} & =1-\frac{u^{4}}{u_{h}^{4}}+a^{2} \hat{\mathcal{F}}_{2}(u), \\
\mathcal{B} & =1+a^{2} \hat{\mathcal{B}}_{2}(u), \\
\phi & =a^{2} \hat{\phi}_{2}(u),
\end{aligned}
$$

where

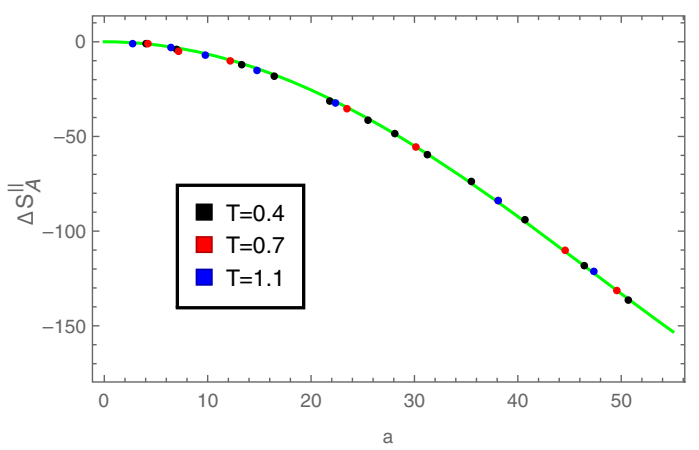

FIG. 5. $\Delta S_{A}^{\|}$in terms of (a) $\frac{a}{T}$ (left) and (b) $a$ (right) at fixed temperatures for $l=0.1$. The green curves show the fitted functions. 


$$
\begin{aligned}
\hat{\mathcal{F}}_{2}(u)= & \frac{1}{24 u_{h}^{2}}\left(8 u^{2}\left(u_{h}^{2}-u^{2}\right)-10 u^{4} \log 2\right. \\
& \left.+\left(3 u_{h}^{4}+7 u^{4}\right) \log \left(1+\frac{u^{2}}{u_{h}^{2}}\right)\right), \\
\hat{\mathcal{B}}_{2}(u)= & -\frac{u_{h}^{2}}{24}\left(\frac{10 u^{2}}{u_{h}^{2}+u^{2}}+\log \left(1+\frac{u^{2}}{u_{h}^{2}}\right)\right), \\
\hat{\phi}_{2}(u)= & -\frac{u_{h}^{2}}{4} \log \left(1+\frac{u^{2}}{u_{h}^{2}}\right) .
\end{aligned}
$$

The temperature and entropy density is then obtained as

$$
\begin{aligned}
T & =\frac{1}{\pi u_{h}}+\frac{(5 \log 2-2) u_{h}}{48 \pi^{2}} a^{2}, \\
s & =\frac{\pi^{2} N_{c}^{2} T^{3}}{2}+\frac{N_{c}^{2} T}{16} a^{2} .
\end{aligned}
$$

Using (9) or (10a), (13), and (23), and after a simple calculation, it turns out

$$
\begin{aligned}
S_{A}^{\perp} & =\frac{V_{d-1}}{2 G_{N}^{d+2}} \int_{0}^{u_{*}} \frac{\left(1-\frac{5}{2} a^{2} \hat{\phi}_{2}(u)\right)\left(1-\frac{1}{2} \frac{\left.a^{2} \frac{\hat{\mathcal{F}}_{2}(u)}{1-\frac{u^{4}}{u_{h}^{4}}}\right)\left(1+\frac{5}{4} a^{2} \frac{\hat{\phi}_{2}(u) u_{*}^{6}-\hat{\phi}_{2}\left(u_{*}\right) u^{6}}{u_{*}^{6}-u^{6}}\right)}{\sqrt{1-\frac{u^{4}}{u_{h}^{4}}} \sqrt{u^{-6}-u_{*}^{-6}}} d u,\right.}{}=S_{A}^{0}+\frac{V_{d-1}}{2 G_{N}^{d+2}} a^{2} \int_{0}^{\hat{u}_{*}} \frac{-\frac{5}{2} \hat{\phi}_{2}(u)-\frac{1}{2} \frac{\hat{\mathcal{F}}_{2}(u)}{1-\frac{u^{4}}{\hat{u}_{h}^{4}}}+\frac{5}{4} \frac{\hat{\phi}_{2}(u) \hat{u}_{*}^{6}-\hat{\phi}_{2}\left(\hat{u}_{*}\right) u^{6}}{\hat{u}_{*}^{6}-u^{6}}}{\sqrt{1-\frac{u^{4}}{\hat{u}_{h}^{4}}} \sqrt{u^{-6}-\hat{u}_{*}^{-6}}} d u,
\end{aligned}
$$

or in the parallel case,

$$
S_{A}^{\|}=S_{A}^{0}+\frac{V_{d-1}}{2 G_{N}^{d+2}} a^{2} \int_{0}^{\hat{u}_{*}} \frac{-2 \hat{\phi}_{2}(u)-\frac{1}{2} \frac{\hat{\mathcal{F}}_{2}(u)}{1-\frac{u^{4}}{\hat{u}_{h}^{4}}}+\frac{5}{4} \frac{\hat{\phi}_{2}(u) \hat{u}_{*}^{6}-\hat{u}_{2}\left(\hat{u}_{*}\right) u^{6}}{\hat{u}_{*}^{6}-u^{6}}}{\sqrt{1-\frac{u^{4}}{\hat{u}_{h}^{4}}} \sqrt{u^{-6}-\hat{u}_{*}^{-6}}} d u .
$$

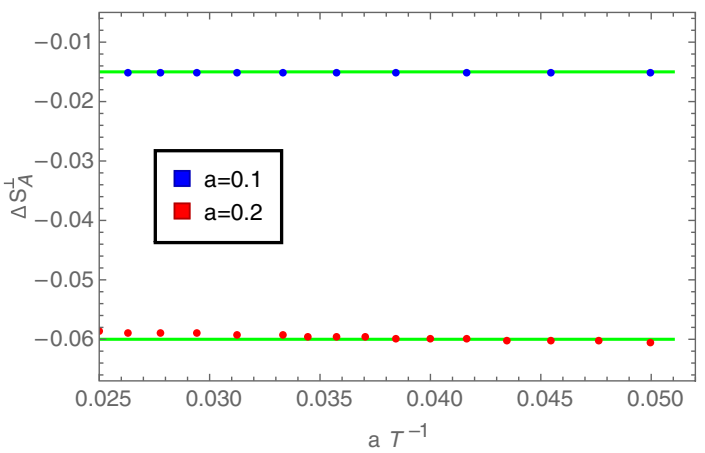

FIG. 6. $\Delta S_{A}^{\perp}$, introduced in (26b), in terms of $a / T$ for $l=0.1$.

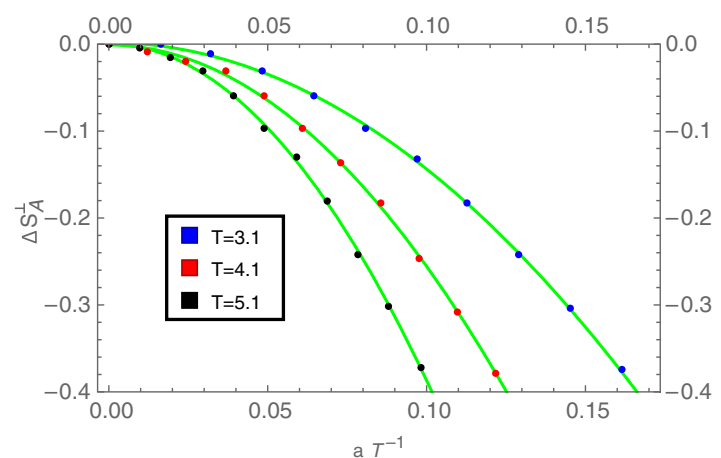

It is important to note that $u_{*}$ in (26a) denotes the turning point of the extremal surface $\gamma_{A}$ (see Fig. 1) in the anisotropic background which is not clearly equal to the corresponding one in the isotropic case, that is $\hat{u}_{*}$. However, since our results indicate that their difference, i.e., $\max \left(\frac{\left|u_{*}-\hat{u}_{*}\right|}{\hat{u}_{*}}\right) \sim 10^{-4}$, is so small, it is a good approximation to neglect this difference, and we therefore replace $u_{*}$ by $\hat{u}_{*}$ in (26b). The same argument is also valid for $u_{h}$ and $\hat{u}_{h}$ corresponding to the horizon radius in the anisotropic and isotropic background. Then the term which is independent of $a$ in (26a) reduces to the entanglement entropy in the isotropic field theory, that is the first term

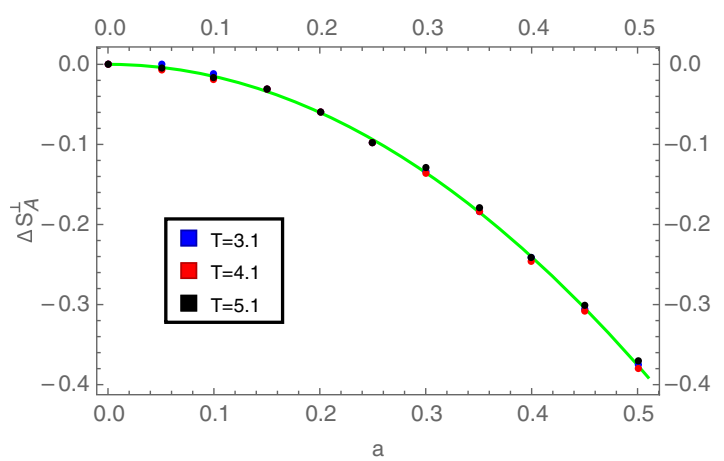

FIG. 7. $\Delta S_{A}^{\perp}$ as a function of (a) $a T^{-1}$ (right) and (b) $a$ (left) in the high temperature limit for $l=0.1$. The green curves show fitted functions. 
in (26b). As a result, the first term in (26b) is independent of an anisotropy parameter and is equal to the entanglement entropy in the isotropic field theory. Although the second term in (26b) explicitly depends on the $u_{h}$ (or equivalently $\hat{u}_{h}$ ), this term, which is equal to $\Delta S_{A}^{\perp}$ according to (12a), is almost independent of the temperature as it is supported by Fig. 6. We can continue the same discussion for the parallel case to find (27). Briefly, in the high temperature limit, the entanglement entropy for both the parallel and perpendicular cases decomposes into two parts: the first (second) part is (almost) independent of an anisotropy parameter (temperature) in agreement with our previous results. In contrast to the general case, in the high temperature limit, Fig. 7 shows that $S_{A}^{\perp}<S_{A}^{0}$. Unfortunately, we cannot find the entanglement entropy between two regions we have discussed here and in the previous section. However, our results indicate that at a fixed temperature, the entanglement must change sign at a specified value of the anisotropy parameter. Similar behavior has been also observed in [8]. For the parallel case, opposite to the perpendicular case, the entanglement entropy is always smaller than the isotropic one. In the high temperature limit, the difference between $\Delta S_{A}^{\perp}$ and $\Delta S_{A}^{\|}$is negligible, and therefore, the figure that resulted for the parallel case is similar to the Fig. 7, and we do not repeat them here.

\section{ACKNOWLEDGMENTS}

M. A. would like to thank the School of Physics of Institute for the research in fundamental sciences (IPM), for the research facilities, and the environment. M. A. would also like to thank H. Ebrahim, M. R. Mohammadi Mozaffar, and M. M. sheikh Jabbari for useful comments.
[1] J. M. Maldacena, The large N limit of superconformal field theories and supergravity, Adv. Theor. Math. Phys. 2, 231 (1998); Int. J. Theor. Phys. 38, 1113 (1999); S. S. Gubser, I. R. Klebanov, and A. M. Polyakov, Gauge theory correlators from non-critical string theory, Phys. Lett. B 428, 105 (1998); E. Witten, Anti-de Sitter space and holography, Adv. Theor. Math. Phys. 2, 253 (1998).

[2] J. Casalderrey-Solana, H. Liu, D. Mateos, K. Rajagopal, and U. A. Wiedemann, Gauge/String Duality, Hot QCD and Heavy Ion Collisions (Cambridge University Press, Cambridge, UK, 2014).

[3] S. Ryu and T. Takayanagi, Aspects of holographic entanglement entropy, J. High Energy Phys. 08 (2006) 045.

[4] D. Mateos and D. Trancanelli, Thermodynamics and instabilities of a strongly coupled anisotropic plasma, J. High Energy Phys. 07 (2011) 054.

[5] M. Jonker, Entanglement entropy of coupled harmonic oscillators, Master's thesis, https://web.science.uu.nl/ITF/ Teaching/2016/2016Jonker.pdf.

[6] M. Headrick and T. Takayanagi, A holographic proof of the strong subadditivity of entanglement entropy, Phys. Rev. D 76, 106013 (2007); M. Headrick, Entanglement Renyi entropies in holographic theories, Phys. Rev. D 82, 126010 (2010); M. R. Mohammadi Mozaffar and A. Mollabashi, Entanglement in Lifshitz-type quantum field theories, J. High Energy Phys. 07 (2017) 120; M. R. Mohammadi Mozaffar, A. Mollabashi, M. M. Sheikh-Jabbari, and M. H. Vahidinia, Holographic entanglement entropy, field redefinition invariance and higher derivative gravity theories, Phys. Rev. D 94, 046002 (2016); M. R. Mohammadi Mozaffar, A. Mollabashi, and F. Omidi, Holographic mutual information for singular surfaces, J. High Energy Phys. 12 (2015) 082.

[7] M. Ali-Akbari and D. Allahbakhshi, Meson life time in the anisotropic quark-gluon plasma, J. High Energy Phys. 06
(2014) 115; M. Ali-Akbari and S. F. Taghavi, Chiral magnetic effect in the anisotropic quark-gluon plasma, J. High Energy Phys. 04 (2015) 181; M. Ali-Akbari and H. Ebrahim, Chiral symmetry breaking: To probe anisotropy and magnetic field in quark-gluon plasma, Phys. Rev. D 89, 065029 (2014); K. Bitaghsir Fadafan and R. Morad, Jets in a strongly coupled anisotropic plasma, Eur. Phys. J. C 78, 16 (2018); K. Bitaghsir Fadafan, D. Giataganas, and H. Soltanpanahi, The imaginary part of the static potential in strongly coupled anisotropic plasma, J. High Energy Phys. 11 (2013) 107; M. Chernicoff, D. Fernandez, D. Mateos, and D. Trancanelli, Drag force in a strongly coupled anisotropic plasma, J. High Energy Phys. 08 (2012) 100; M. Chernicoff, D. Fernandez, D. Mateos, and D. Trancanelli, Jet quenching in a strongly coupled anisotropic plasma, J. High Energy Phys. 08 (2012) 041; S. Chakraborty and N. Haque, Holographic quarkantiquark potential in hot, anisotropic Yang-Mills plasma, Nucl. Phys. B874, 821 (2013); D. Giataganas, Observables in strongly coupled anisotropic theories, Proc Sci., Corfu2012 (2013) 122; D. Giataganas, Probing strongly coupled anisotropic plasma, J. High Energy Phys. 07 (2012) 031; V. Jahnke, A. Luna, L. Patio, and D. Trancanelli, More on thermal probes of a strongly coupled anisotropic plasma, J. High Energy Phys. 01 (2014) 149; L. Patino and D. Trancanelli, Thermal photon production in a strongly coupled anisotropic plasma, J. High Energy Phys. 02 (2013) 154; D. vila, D. Fernndez, L. Patio, and D. Trancanelli, Thermodynamics of anisotropic branes, J. High Energy Phys. 11 (2016) 132; R. Mishra and H. Singh, Entanglement asymmetry for boosted black branes and the bound, Int. J. Mod. Phys. A 32, 1750091 (2017).

[8] M. Rahimi, M. Ali-Akbari, and M. Lezgi, Entanglement entropy in a non-conformal background, Phys. Lett. B 771, 583 (2017). 